\title{
SCIENTIFIC REPRTS \\ OPEN Author Correction: Multiplexed imaging of high-density libraries of RNAs with MERFISH and expansion microscopy
}

